\title{
Guidance for Behavior Analysts in Addressing Racial Implicit Bias
}

\author{
Catherine Jaramillo and Karen Nohelty* \\ Center for Autism and Related Disorders
}

Center for Autism and Related Disorders, 21600 Oxnard Street, Suite 1800, Woodland Hills, California, 91367, United States

* Corresponding Author

Karen Nohelty, M.Ed.

Center for Autism and Related Disorders

k.nohelty@centerforautism.com

(562) 999-7788 


\begin{abstract}
In the practice of applied behavior analysis (ABA) treatment, implicit bias, which can be defined in behavioral terms, needs to be addressed as it may inadvertently lead to overt forms of discrimination on the basis of race. While little research has been conducted within the field of ABA on racial implicit bias, information gathered from related fields can provide insight as to how behavior analysts can promote positive change in this area. Drawing from existing literature, recommendations are provided regarding assessment and administration of interventions to reduce racial implicit bias for clinicians. The purpose of this paper is to provide strategies that behavior analysts can implement immediately to assess and reduce behaviors related to implicit bias exhibited by practitioners, thereby reducing racial discrimination with clients and staff.

Keywords: implicit bias, discrimination, race, training, applied behavior analysis
\end{abstract}


This manuscript is being published on an expedited basis, as part of a series of emergency publications designed to help practitioners of applied behavior analysis take immediate action to address police brutality and systemic racism. The journal would like to especially thank Associate Editor, Dr. Jomella Watson-Thompson. Additionally the journal extends thanks to Janani Vaidya and Dr. Marlesha Bell for their insightful and expeditious reviews of this manuscript. The views and strategies suggested by the articles in this series do not represent the positions of the Association for Behavior Analysis, International or Springer Nature.

Guest Editor, Denisha Gingles 


\section{Guidance for Behavior Analysts in Addressing Racial Implicit Bias}

Systemic racism has been a part of our society for generations. However, over the past decade, the conversation around implicit bias has grown in corporate settings and in the mainstream (Kempf, 2020). Most notably, the deaths of Breonna Taylor and George Floyd in 2020 ignited protests, discourse, and movements (i.e., Black Lives Matter) around both explicit and implicit racism in the United States. These egregious acts, however, are unfortunately not the only ones that have occurred recently. In a report released by the FBI, hate crimes in the United States were found to increase by 3\% in 2019 and the majority of these crimes (57.6\%) were targeting individuals because of race or ethnicity (U.S. Department of Justice, Federal Bureau of Investigation, 2020). While these overt acts of racism are horrific and urgently need to be addressed, this paper focuses on a more subtle form of racism known as implicit bias, which is prevalent across societal institutions including behavioral health care services. This paper suggests that the field of applied behavior analysis ( $\mathrm{ABA}$ ) has immediate interventions that can be used to reduce implicit biases within our practice.

Implicit bias has traditionally been considered an unconscious process where underlying attitudes and stereotypes cause outward behaviors that are not intended by the individual to be biased. However, as asserted by De Houwer (2019), implicit bias is a behavioral phenomenon, defined as behavior that is influenced by cues of another's membership in a particular social group (e.g., race) in a manner that is quick, unintentional, or difficult for the individual to control. An analysis of direct contingencies (e.g., respondent conditioning, operant condition, 
observational learning, stimulus generalization) can explain the presence of racism and bias (Matsuda et al., 2020). Additionally, a growing body of work in the area of relational frame theory (RFT) has led to a behavior analytic explanation of bias and racism based on derived relational responding (DRR). As discussion of RFT is beyond the scope of this paper, the interested reader is referred to Critchfield and colleagues (2018) for a description of DRR and to Dixon and colleagues (2003) for an analysis of terrorism and prejudice from an RFT perspective. When viewed as an unconscious or unintentional process, implicit bias may not only negate personal accountability, but it seems to be outside the scope of ABA. However, analyzing implicit bias through a behavioral lens has the potential to lead to socially meaningful behavior change, which may be harnessed by ABA clinicians.

In the provision of medical services, racial biases held by clinicians may impact patient care. Some studies in the medical field indicate that implicit bias on the part of the clinician can lead to negative effects for patients; however, more research is needed to confirm this impact (Zestcott et al., 2016). Specifically, in a study with children with autism spectrum disorder (ASD) and other developmental disabilities, Magaña and colleagues (2012) found that Black and Latino children were more likely to score lower on caregiver reports of health care quality. Furthermore, ethnic minority children with ASD receive their diagnosis and start intervention at an older age than white children, and they less frequently receive interventions that are evidence based (Roche et al., 2018). While more research is needed to identify why this delay occurs for BIPOC (Black, Indigenous, and people of color) children, the lack of a biological or genetic diagnostic test results in the need for clinicians to heavily rely on observations, clinical judgment, and clinical interviews, which can be influenced by implicit bias on the part of the clinician. Additionally, the ability to build rapport between clinician and client can be harmed by 
implicit bias on the part of the clinician, even in the absence of explicit bias (Kanter et al., 2020); ostensibly this rapport would be harmed by implicit bias on the part of the client as well.

Clinicians implementing ABA are likely also influenced by implicit bias. In fact, a systematic review completed by FitzGerald and Hurst (2017) indicated that physicians and nurses demonstrated implicit bias at levels similar to the general population. Ignoring implicit bias does not mean it does not exist; given this information about physicians and nurses, clinicians working in the field of ABA cannot assume that they do not hold implicit biases. Specifically, being present in an environment where one culture dominates decision making (which in the United States is the Anglo-European culture) can lead to a lack of awareness of the surrounding cultural and racial contingencies, which can result in potentially unintentional "behavioral missteps" (Tagg, 2020). While culture and race are not synonymous, they are interrelated (Capell \& Sevon, 2020). However, it is critical to avoid assumptions that all individuals who identify of the same race share the same cultural practices. At the same time, it is important to recognize that race is one of the most salient visual characteristics of an individual.

Demographic data collected by the Behavior Analyst Certification Board (BACB, n.d.) indicate that several racial groups (i.e., Black, American Indian/Alaska Native, Hispanic/Latinx) are underrepresented as Board Certified Behavior Analysts (BCBAs) and Board Certified Behavior Analysts-Doctoral (BCBA-Ds) when compared to the general population (U.S. Census Bureau, 2019). Thus, it is likely that BIPOC clients will be served by white clinicians. In order to provide the best care for all individuals, a better understanding of the extent and impact of clinicians' implicit bias is needed. The Professional and Ethical Compliance Code for Behavior Analysts (BACB, 2014) contains several items supporting the need for clinicians to address 
implicit bias in their practice (i.e., 1.01 "reliance on scientific knowledge,"1.02 "boundaries of competence," 1.05 "professional and scientific relationships").

By focusing on observable, measurable behaviors related to implicit bias, ABA interventions have the potential to change what people do in order to improve client outcomes. De Houwer (2019) described implicit bias as "something that people do rather than something that people possess;" when implicit bias is discussed as an "unconscious" process, it can be viewed as something that cannot be changed or lead to defensiveness on the part of the individual. Viewing implicit bias from a behavior analytic perspective could lead to reduced controversy and increased openness to addressing it on the part of clinicians. Committing to providing the best care for clients involves assessing the presence of racist behavior, including overt actions and private behaviors, and addressing any that exists.

The purpose of this paper is to offer practical tools that clinicians can implement immediately in order to address implicit bias and reduce resulting discrimination as it relates to race. First, this paper addresses the assessment of implicit bias. Existing literature is reviewed, and recommendations are provided for assessing a clinician's implicit bias during client, caregiver, and staff interactions. Next, interventions to target the reduction of implicit bias are discussed. As there is limited relevant work in the field of ABA, information is drawn from existing literature, and methods to reduce biased behaviors. Regardless of setting (e.g., clinic, community, home, school, group homes, residential facilities), the strategies discussed involve common activities that a behavior analyst may engage in.

\section{Assessment of Implicit Bias}

\section{Literature Review}


While the study of implicit bias has not yet been widely adopted within the field of ABA interventions, other related fields have developed measurement tools. One such assessment tool is the Implicit Association Test (IAT), which was derived from a social-cognitive perspective, has been cited over 10,000 times, and has been the focus of multiple meta-analyses (Kurdi et al., 2018). Currently, the primary form of the IAT is a computerized task, which can target comparison of associations by measuring response latencies and accuracy. A word or image flashes (e.g., picture of a Black woman, picture of a white woman) and the respondent presses the key corresponding to one of two categories (e.g., good, bad); the duration of time to respond to various presentations of comparisons is scored. Stronger associations between two constructs are demonstrated by shorter latencies and fewer errors on the test (Kurdi et al., 2018). Since it was first created, the IAT has been used across a number of fields to determine the strength between presented associations of constructs, such as physical size, sexual orientation, and gender because these biases are otherwise potentially obscured by self-report (Steffens, 2004). The IAT has most notably been used for determining racial biases due to the social impact of this particular type of bias.

A newer measurement tool, the Implicit Relational Assessment Procedure (IRAP), was developed within the fields of social science and behavior analysis (Barnes-Holmes et al., 2010). The IRAP measures implicit bias as a relational behavior rather than an associative activity. Therefore, it looks at stimulus equivalence as a way to link the history of conditioning of cultural norms to derive implicit biases. Like the IAT, the unit of measure can be latency or accuracy. However, these are measured between "blocks" that are either consistent or inconsistent with cultural norms, rather than between dyads of constructs such as "Black and white" or "good or 
bad." The IRAP therefore relates a behavior (response time) to previous exposure to the particular equivalences or social constructs that are being presented.

In determining which of these measurement tools may be useful in data collection for ABA clinicians, it is beneficial to look at their respective strengths and weaknesses. The IAT has not demonstrated strong sensitivity at detecting individual attempts to hide biases, unless the participants have been specifically told to go slower (Kim, 2003). While the participants may skew their results leading to higher errors, they are not able to obscure the shorter latencies as a result of their implicit associations (Steffens, 2004). However, when participants have had previous exposure and experience with the IAT, skewing of results is both more likely and leads to a higher effect size (Steffens, 2004). This should be of concern if used as a repeated measure to determine if an intervention, when applied, may have an effect on the implicit biases of the participants. While the IAT may provide researchers a way to infer biases based on the associations that are recorded from the participant's responses, it fails as a measurement tool in its lack of information on the directionality of the associations (Power et al., 2009). This limited information provided by the IAT is therefore not sufficient in providing a baseline for identifying behaviors to target for change in decreasing implicit bias.

The IRAP, while not yet as widely used, was born from a behavior analytic explanation of cognition, RFT (Barnes-Holmes et al., 2010). It has been studied to confirm both its validity and reliability in evaluation of race-based implicit biases (Drake et al., 2015). The IRAP can provide more nuanced and complete information in comparison to the IAT. For example, Drake and colleagues (2015) suggest differences in biases between in-group (Black responses for proor anti-Black options) and out-group responses (Black responses for pro- or anti-white options), a finding that was not captured with the IAT. This points to implicit biases as a reflection of 
Implicit Bias 10

preference for the group that the individual belongs to, rather than a reflection of negative beliefs towards the group to which they do not belong. The additional information that the IRAP provides, such as the bidirectionality of the associations, may be of particular interest to behavior analysts. However, it is also important to consider that further research is needed. For example, a recent study has identified a "generic pattern," which points to weak stimulus control from the category stimuli over response times. This suggests that the IRAP may not be as sensitive to the learning histories that it claims to measure (Hussey \& Drake, 2020). Therefore, the IAT and IRAP alone do not provide information on how to intervene or change behaviors related to implicit bias.

\section{Recommendations for Clinicians}

While the IAT and IRAP may be used by clinicians to identify training needs, neither are currently sufficient as stand-alone measures and repeated presentations are problematic. While research does not point to the predictability of behavior as a result of scores on the IAT or IRAP (Meissner et al., 2019), analysis of situations in which implicit bias is common can help to identify behaviors for change. Racially biased behaviors are those that occur as a result of an individual's race and they are considered implicit when they occur unintentionally. Due to the unconscious nature of these behaviors, ABA clinicians would likely be unaware of any occurrence. Therefore, collecting data on specific behaviors can help clinicians identify situations in which they are responding quickly and effortlessly on the basis of the social cue of race or, in other words, in an implicitly biased manner. Focusing on specific, socially significant behaviors may provide more valuable information to a clinician in assessing implicit bias than the results of the IAT or IRAP. 
Data collected during clinicians' interactions with clients, caregivers, and staff of different races may be used to identify underlying implicit bias. In this section, methods to administer evaluations of implicit bias are discussed. In addition, examples of measurable behaviors that may indicate implicit bias are provided.

\section{Administration}

Evaluations of implicit bias may be carried out by another clinician (as peer or supervisor feedback) or by the clinician themselves during their own interactions with clients or staff (as a self-evaluation). Self-evaluation has the benefit of not requiring others for administration and enables private events to be addressed (e.g., anxious, avoidant, or stereotypic thoughts in various situations with those of other races). Additionally, less empirical reflection may be beneficial. For example, self-reflection on one's own culture, values, beliefs, characteristics, preconceived notions, and background experience can be an important step in assessing one's potential sources of bias (Fong et al., 2016); this identification is the first step towards ensuring that those factors are not pushed onto others unconsciously or inappropriately. In addition to potential selfassessment tools presented by Fong and colleagues (2016; i.e., "Diversity Self-Assessment," "How Do You Relate to Various Groups of People in Society," "Multicultural Sensitivity Scale"), Tagg (2020) describes the ADDRESSING framework, which is a model for assessing one's own culture as well as the culture of others, one part of which addresses race and ethnicity. After self-reflection, discussion with those from backgrounds other than one's own can help gain additional perspectives to aid further self-evaluation. This should be an ongoing, iterative process approached with a flexible, open-to-change mindset.

Whether assessments are performed by other clinicians or via self-evaluation, collected data can be compared across client, caregiver, and staff interactions to assess any differences in 
clinician behavior across individuals of different races, which could indicate a functional relationship between the individual's race and the clinician's behavior. However, it should be noted that in order to provide equitable services, that may mean the provision of a given behavior in differing rates across clients; for example, some clients/caregivers may need more or less of a given strategy in order to meet their individual needs.

\section{Measurable Behaviors}

In the below sections, examples of behaviors potentially influenced by race are detailed to support clinicians in operationally defining, measuring, evaluating, and intervening on them (potential intervention methodologies are detailed in a later section). This is not intended to be a comprehensive measurement of implicit bias, but rather a list of behaviors that may be measured and analyzed on an individual basis, with a clinician selecting one or two behaviors at a time to assess. Specific emphasis is given below to behaviors that could be measured in the natural environment, to address a limitation of tests such as the IAT and IRAP. Specific operational definitions should be developed for each context in which they are used. Additionally, as culture and race are often intertwined, to address the individualized nature of ABA services, culture is included in some of the below examples as an identification of individualized expressions that may be related to race. When working with individuals, thinking in terms of individual culture instead of broader race forces practitioners to ask questions instead of making assumptions. However, clinicians should consider both culture and race to avoid missing critical parts of an individual's identity and experience. Examples are provided across contexts in which clinicians interact with clients and staff during the provision of services, including: (a) intake; (b) direct client interactions; (c) caregiver interactions; and (d) staff interactions. See Table 1 for specific information regarding contexts, operational definitions, and examples for each. 
Intake. The first introduction to a client (and their caregiver, in the case of a minor or conserved individual) is typically during an intake or initial assessment meeting (this may be named differently depending on the setting, for example, in the school setting this may be a parent-teacher conference). This intake process is an ideal time to gather data and lay the foundation for an appropriate working relationship that considers the individual's race and culture. Implicit biases held by clinicians may impact their behavior during this intake process that can have repercussions throughout intervention. Specific areas in which data can be collected during the intake process to reveal potentially implicitly biased behavior include: (a) gathering detail regarding race/culture on intake/assessment paperwork; (b) asking relevant questions about cultural preferences; (c) acknowledging and incorporating the response regarding the client's preferences; and (d) incorporating a behavior into a treatment plan.

Intake assessments and related paperwork could be assessed in relation to the detail they provide regarding the family's race and cultural preferences (i.e., using permanent product data collection). In the absence of this information, clinicians are unable to assess differences in their behavior based on the race and culture of their client and are potentially more likely to engage in behaviors controlled by salient visual cues (e.g., assumed racial group) as opposed to individualized client needs. Data could be collected on the number of items/questions that address racial/cultural differences included in the intake paperwork. Another critical way of gathering information is by asking questions about cultural preferences (either of the client directly or of the client's caregiver). For example, knowledge of a family's preferred sleeping practices (e.g., co-sleeping, bedtime) can inform culturally relevant interventions for sleep problems. There are several components that could be operationally defined in this area, such as avoiding making assumptions, asking open-ended questions instead of close-ended questions that 
lead the family to a specific response, and asking questions that are directly related to relevant aspects of the family's culture. The number of questions asked by a clinician meeting these criteria during an intake meeting could be tracked as well. Differences in these clinician behaviors across clients of different races could indicate implicit bias.

Acknowledging the information provided by the client/caregiver can aid in building rapport and communicating understanding of the client's needs and concerns. Some key components of this skill include avoiding making statements of judgement, responding with supportive acknowledgement, and following up on concerns stated by the client/caregiver. The intake process typically ends with the completion of a treatment plan. The first step of incorporating a behavior (whether targeted for increase or decrease) into a treatment plan is to identify if it is appropriate for treatment. BCBAs are ethically bound to incorporate clients into the "planning of and consent for behavior-change programs" (BACB, 2014). Taking this a step further to explicitly ask about the client's cultural preferences is an additional step toward reducing bias. Data could be collected on the frequency with which the clinician seeks input from the client/caregiver regarding inclusion of a goal or strategy within the treatment plan. Race and culture can play a part in many potential goal areas, including sleeping, dressing, feeding, making eye contact, greeting others, observing holidays, toileting, and behavior intervention plans. Gathering detailed input from the client/caregiver can aid the clinician in avoiding making implicitly biased treatment decisions and ensuring that all clients receive individualized treatment plans regardless of their race.

Direct Client Interactions. Implicit bias has been studied in direct client care, with evidence indicating that "white providers who score higher on measures of implicit bias, but not explicit bias, speak faster, dominate conversations, have shorter visits, display fewer positive 
Implicit Bias 15

nonverbal cues and less warmth, and use more first-person plural pronouns and anxiety related words when interacting with Black patients" (Kanter et al., 2020). This previous research provides insight into potential behaviors related to implicit bias that could be operationally defined and tracked. Categories of potential data collection include: (a) vocal behavior, (b) nonvocal behavior, (c) stimuli, (d) language of instruction, and (e) response to explicit racial challenges.

A clinician's choice of words with a client can reflect implicit bias and has the potential to lead to differential care on the basis of race. Frequency data could be collected on these verbal behaviors such as statements of praise and corrections/redirections during direct sessions with clients. Rate of speech and tone of voice are additional vocal behaviors that may be indicative of implicit bias that may be more challenging to operationally define and/or record; however, there may be situations where taking the time to define and develop a data collection system for these behaviors would yield meaningful information.

Nonvocal behavior is also critical to our social interactions with others. Use of restraint or other physical procedures (e.g., use of full physical prompts) could be tracked using frequency data; the differentially greater use of such procedures with individuals of a given race would clearly call for additional follow-up. Additionally, more subtle nonvocal behavior such as eye contact, facial expressions (e.g., smiling), and posture (e.g., crossing arms versus a more relaxed posture) could be assessed. The provision of physical reinforcers to clients (as well as the duration provided) is another aspect that could be tracked.

During sessions or teaching interactions, the clinician's choice of stimuli (e.g., pictures, books, videos, art supplies) to teach targeted skills can expose implicit bias. Representation of individuals from diverse backgrounds could be scored in the stimuli used for a given client. 
While it may be difficult to determine a set criterion regarding the amount of diversity appropriate in stimuli for a given client, the Anti-Bias Curriculum (2001) provides guidelines related to classroom settings that could be extrapolated to other settings as well: "token" diversity should be avoided regardless of the client's race; stimuli should include those from the client's race as well as from other diverse backgrounds (important even for white children); and stereotypic images should be avoided (e.g., a Native American in a headdress).

For any client whose native language is not English, methods of incorporating their native language should, at a minimum, be considered. For example, not only does the evidence demonstrate that children with ASD raised in bilingual environments do not have additional language delays (Drysdale et al., 2015), but also several studies indicate improved child performance when intervention is provided in their native language (Lang et al., 2011, Lim \& Charlop, 2018). While instruction in English may be part of standard practice, assessing if standard practice is appropriate or could potentially be modified to the benefit of the client is a step toward improving client care by reducing potential bias and discrimination as well as creating a more inclusive setting.

An additional scenario that may arise during direct sessions with clients is an explicit racial challenge. This may come from a staff member (e.g., "Why don't their parents keep their hair cut short, it would be so much easier to manage"), from another client/child (e.g., "Why is his hair long?"), or from the client themselves (e.g., "Why does the band-aid match your skin but not mine?"). Clinicians may not have received training in responding to racial challenges and may ignore to avoid confrontation. However, the Anti-Bias Curriculum (2001) again provides some guidance in how to respond to challenges from a young child; it is important to avoid ignoring, changing the subject, answering indirectly, or criticizing identification of physical 
differences, and instead the clinician should answer questions and respond to situations factually. Kanter and colleagues (2020) provide a scoring system for rating physician responses to racial challenges, ranging from a score of "strong, immediate negative reaction" to "strong, immediate positive reaction." This scoring system could be used to collect data on clinician's responses to racial challenges during direct client interactions. See Table 2 for a modified version of this scoring system with examples relevant to the provision of $\mathrm{ABA}$ services.

Caregiver Interactions. Often caregivers are involved in their child's intervention. Reducing behaviors related to implicit bias has the potential to improve the therapeutic relationship between the clinician and caregiver, which is a critical component of client care and has the potential to improve client outcomes (Taylor et al., 2018). Related behaviors to consider assessing include: (a) using verbal microaggressions; (b) making assumptions instead of asking questions; (c) using reinforcing/positive statements; (d) incorporating collaborative versus authoritative strategies; (e) considering extended family members as potential caregivers; (f) considering caregiver's preferred language; and (g) using evidenced-based methods of assessing and training.

Microaggressions related to race can be described as "brief, unconsciously denigrating messages to people of color in the form of subtle snubs or dismissive looks, gestures, and conversational tones during everyday exchanges" (Fong et al., 2017). Microaggressions can range from statements that are rude or demeaning to those that are more subtle. An individual who engages in a microaggression may not be aware of the harmful impact of their words on the other person. Therefore, self-evaluation of this behavior may lead to an underestimate; as such, it is recommended that a peer or supervisor, preferably an individual from the same race/culture as the caregiver, track data on microaggressions. As individual perceptions vary regarding what 
constitutes a microaggression, ideally the person the microaggression is aimed toward would identify if one occurred; however, this may be challenging, depending the therapeutic alliance between the individual and the caregiver.

Additionally, data could be collected on making assumptions instead of asking questions, as assumptions may reflect the clinician's own values or culture and could be used more frequently with those of certain races. Inaccurate assumptions related to caregiver priorities or past experiences could be detrimental to client outcomes. A clinician's use of positive phrases with a caregiver (assumed to function as reinforcers) could be tracked. During caregiver interactions with the client, this may involve responding to the caregiver's appropriate use of a strategy. The specific behavior identified for reinforcement during a consultation without the client may vary; potential caregiver behaviors to reinforce include sharing successes (e.g., a positive story of an interaction with their child) and describing a situation where they used a specific strategy.

Brookman-Frazee (2004) demonstrated that the use of collaborative strategies within a caregiver education program led to more positive outcomes for both the caregivers and their children than the use of clinician-directed strategies. Given this, assessing the use of collaborative versus authoritative strategies may be a critical step in providing best practices to clients and caregivers of all races. Examples of collaborative strategies include providing choices, asking open-ended questions (in lieu of close-ended questions), providing suggestions instead of directives, and involving the caregiver in decision making. Additionally, broadening the consideration of who is a caregiver beyond the client's parents serves to include those of cultures who traditionally place a greater value on extended family members. Even if other 
Implicit Bias 19

family members are not caregivers of the client, their views and values may impact the parent's decision making and response to proposed interventions.

The language of clinician-caregiver interaction has the potential to impact the provision of services. In an assessment of children with ASD, Zuckerman and colleagues (2017) found that caregivers with limited English proficiency were less likely to be knowledgeable about ASD and had children receiving fewer therapy hours. While some caregivers may speak the clinician's native language, consideration should be given to language of choice for the caregiver; to avoid making assumptions regarding which caregivers speak a second language, all caregivers could be asked about language preference. While collaborating with caregivers in the clinician's native language may be ultimately preferred by the caregiver, making an assumption without caregiver input could be a sign of bias and impact the clinician-caregiver relationship.

Additionally, the clinician's assessment of a caregiver's proficiency in use of interventions with their child and decision to use evidence-based strategies such as behavioral skills training (BST) during meetings may vary depending on a quick, unconscious assessment of social cues (e.g., the caregiver's proficiency with the clinician's native language, the caregiver's race). Data on the number of opportunities evidence-based strategies, such as BST, were used would highlight any discrepancies.

Staff Interactions. The interactions ABA clinicians have with their colleagues and the behavior technicians (BTs) they supervise provide another area in which behaviors can be assessed with the goal of identifying potential implicit bias and reducing racial discrimination. Recently collected demographic information from the BACB indicates a racial discrepancy between Registered Behavior Technicians ${ }^{\mathrm{TM}}$ and BCBAs/BCBA-Ds (with $47.63 \%$ and $71.82 \%$ of the groups, respectively, reporting themselves as white; BACB, n.d.). Thus, it is possible that 
a white supervisor will be overseeing a BT who is BIPOC. There are several components of staff interactions to consider, including: 1) staff training; 2) ongoing mentorship; and 3) development of professional goals.

Assessing the presence of racial diversity in trainers can provide information to identify if intervention is necessary. Over-inclusion of models of the same race as those in power at an organization could be a display of implicit bias and have detrimental effects for staff of other races. Data could also be collected on the reaction of supervisors to staff issues; responding differently to those of different races could be an outward behavior related to the social cue of race and not the supervisee's behavior. Possible reactions could include responding punitively (e.g., writing up or reprimanding the employee), ignoring the issue, or responding supportively (e.g., with errorless learning and differential reinforcement). Further, social validity data could be gathered regarding staff's perception of the equitable provision of training opportunities.

During mentorship, the number of mentoring opportunities offered to individuals of different races could be tracked. Additionally, data could be collected on the quality of those mentoring experiences, including many of the items listed above in the caregiver interaction section (e.g., using verbal microaggressions, making assumptions instead of asking questions, using reinforcing/positive statements, and incorporating collaborative versus authoritative strategies). However, one area specific to mentoring is the explicit incorporation of discussions of race and culture into conversations with supervisees. Assessing how often discussions around race are initiated or responded to appropriately by the mentor can be valuable in identifying how well mentorship is addressing this area of development. Specific behaviors a mentor can engage in to support their mentee's development in cultural competence and awareness of implicit bias include modeling comfort/openness in discussing difficult topics, engaging in self-reflection, 
changing position when confronted with new data/information, and admitting mistakes;

modeling these behaviors outside of discussions around race may also serve to increase an overall skill set that will aid the mentee in identifying and responding to potential implicit bias in their own behaviors.

The provision of professional goals is a key element in supporting the development of individual staff members. Supervisors may find it easier to write goals that are related to clinical needs than those surrounding to professionalism (e.g., attitude, assertiveness) as clinical needs may be easier to operationally define and communicate. As such, supervisors may find it awkward or difficult to write professional goals surrounding discrimination or implicit bias as it relates to a staff member's work with individuals and their families. On the other hand, a supervisor may write a professional goal that is under the control of an individual's race as opposed to their job-related behavior. For example, goals related to a supervisee's personal appearance or tone may attempt to target reduction of a behavior that is part of the supervisee's cultural experience. An analysis of professional goals can be conducted to assess their function and social significance, including identifying the specific behavior to be changed (e.g., focusing on behaviors targeted for improvement), why its targeted for change (e.g., related values), and the impact of that change within the workplace. A goal that is written to address a specific client need (e.g., changing one's tone with a specific client due to client sensory sensitivity) has a very different outcome than a goal written without a clearly defined purpose (e.g., changing one's tone overall throughout work contexts). Trends in professional goals targeted across races may illuminate the impact of implicit biases on the part of supervisors.

\section{Interventions to Reduce Implicit Bias}

\section{Literature Review}


Implicit bias trainings have become ubiquitous within mainstream society, highlighting in particular the well-intentioned but missed opportunities by corporations to address racial tensions (Kempf, 2020). While some may think that one-time trainings suffice as interventions, they have instead left a plethora of questions around what we have "right" and what is left to establish as effective behavior change strategies in battling implicit biases. A worthwhile goal within all fields should be to enact protocols that result in socially significant behavior change. While studies are limited, there is a growing body of work that analyzes and proposes suggestions for future trainings. One article described a workshop used to train college campus police (Fernandes, 2016). Lectures were used to explain and structure conversation around biases and why these biases may affect the work and performance of the campus police. One of the instructors noted the difficulty in assessing the effectiveness of the trainings due to the absence of benchmarks; as such, no data were presented in this article. Jackson (2018) provided a review of the 2.5-day training by the Fair and Impartial Policing organization on implicit bias. The article provides an analysis of how this training, in an effort to reduce shame and increase positive feelings from the participants, actually failed to change biased behavior because it decreased the trainee's motivation to take action. Other research-based studies have similarly targeted decreasing implicit biases through various methods, such as by simply providing participants with their IAT results or through implementation of interventions such as the retraining of previously held associations. These studies are a good starting point and provide substantial guidance for next steps.

The way that a respondent relates to their IAT or IRAP results is an important consideration, however, the results alone are not sufficient in reducing implicit biases. Hillard and colleagues (2013) used the IAT as an educational tool and not simply a measure. By 
Implicit Bias 23

providing the IAT results to the college students, they found that those who demonstrated a high preference for European Americans also reported more negative reactions to the IAT and tended to depersonalize their scores. This strongly negative reaction may be related to the undesirable connotations of having biases in the current culture. However, when these same participants received a lost email belonging to an African American, they were also more likely than participants who did not report a high preference for European Americans to engage in helpful behavior by responding to the sender in an effort to rectify the situation. This observation of improved prosocial behaviors without addressing the negative reaction supports the previously stated suggestion by Jackson (2018) in their criticism of current police trainings in that assuaging the participants' guilt may not lead to the target antibias behaviors. Conversely, having the participants be aware of and understand the guilt tied to implicit bias may result in social positive antibias behavior. Hillard and colleagues (2013) recommend the use of the IAT as intended, as a measurement tool. More importantly, they suggest the use of other methods of instruction to inform the participants on implicit biases as well as the use of actual interventions, not just the measure of implicit bias, when the goal is to reduce them. Additional forms of instruction may help to decrease the impact of implicit biases and increase the use of prosocial behavior.

Re-pairing stimuli (Evaluative Conditioning) may be one of the ways in which implicit biases could be retrained so as to alter the negative associations held by an individual (Lai et al., 2013). Lai and colleagues (2013) accomplished this by instructing the participants to re-pair a previously negative association of Black faces with both positive visuals (text) and vocal ("yes") behaviors. This exposure resulted in the decrease of implicit biases; however, the quality of those interactions was not reflective of the participant's behavior outside of the test setting. This study, 
similar to Hillard and colleagues (2013) study, present important steps forward and have provided ideas for continued areas of study.

Additional work is needed to ensure that long-term behavior change occurs. The field of ABA has highlighted the importance of generalization and maintenance of behavior improvement. A strength of the Hillard and colleagues (2013) study is that the participants took the IAT in the classroom as part of consciousness training for how to interact with others of different races and therefore they were both in the presence of those they would typically interact with and in the target location, the university. Similarly, the new associations made in the Lai and colleagues (2013) study were present when measured two days later. Stronger support for maintenance (across an 8-week period) was demonstrated when a rehearsal of intervention strategies was incorporated into the treatment package (Devine, et al., 2012). A recent review found that less than $10 \%$ of published studies on implicit bias included measures of maintenance (Lai et al., 2013). While the Devine and colleagues (2012) study more strongly supports maintenance, like the Hillard and colleagues (2013) study, it also states that information when presented alone regarding the presence of implicit bias is not sufficient to reduce bias on its own. Furthermore, it is unclear as to whether the effects of the Devine and colleagues (2012) study will extend past the specific context of an implicit bias measure and to the application of prosocial behavior in the natural environment. Thus, the above-listed interventions have not expanded these results to empirically establish that the generalization of implicit bias reduction has been extended to natural environments (e.g., a reduction in biased behavior in the appropriate context).

\section{Recommendations for Clinicians}


Implicit Bias 25

For ABA clinicians, maintenance and generalization are critical components when considering interventions to target reduction of implicit bias. Research is limited on implicit bias interventions specifically within ABA. However, there are well-established ABA strategies that are known to successfully alter behaviors across a multitude of domains. In this section, we explore ways in which clinicians can use these ABA strategies to address behaviors related to implicit bias, specific to the intake process, direct client interactions, caregiver interactions, and staff interactions. Reinforcement, self-management, BST, Acceptance and Commitment Training (ACT), and generalization of stimuli are evidence-based strategies that can be implemented now to start the work towards a more aware and anti-bias practice (see Table 3 for a summary of interventions, suggested applications, and research support). However, future research is needed to evaluate the effect of specific intervention protocols on various measures of implicit bias, like those discussed previously.

Reinforcement was one of the first behavior analytic principles studied in the field and continues to be a prevalent strategy. A simple, but not necessarily easy, strategy to implement is to increase the reinforcement contingencies around interpersonal interactions with members of different races. Clinicians can select and provide reinforcement in response to prosocial interactions between clinicians and clients/caregivers during the intake process, targeting the behaviors mentioned in the sections above, such as asking open-ended culturally cognizant questions or including treatment recommendations that have culturally relevant interventions that stem from caregiver input. Similarly, during direct client interactions, clinician vocal and nonvocal behavior, such as the equitable delivery of reinforcers to their clients of different races, can also be targeted for increase. A substantial quality assurance structure should allow for ongoing staff training in order to observe, and then provide reinforcement in response to, these 
Implicit Bias 26

target behaviors. Additionally, reinforcement can be provided for the provision of inclusive recruiting, hiring, and mentoring behaviors.

When such outside oversight is not possible, self-management can be utilized as a way to encourage the practitioners themselves to evaluate their progress in mitigating the implicit biases they carry and related outward behaviors. In fact, there is research to support that individuals may prefer self-management as a way to reflect on their progress. Schultz and Baker (2017) found that $70 \%$ of participants selected a self-management strategy as their preferred method towards identifying and addressing their implicit biases. However, in the absence of component analyses, it is imperative to combine self-management with other interventions, if those additional resources are available. For example, Knochel (2019) successfully used selfmonitoring, in conjunction with focused and written performance feedback, to reduce racial disparity in behavior specific praise delivered in the classroom. A small change in teacher behavior, produced by a multi-component training on equity, led to observable improvements in the classroom, including a reduction in disruptive behaviors and an increase in academic engagement. While this specific finding has not been peer reviewed, it is in line with previous studies on the effectiveness of performance feedback.

BST is a procedure used to teach others a skill that includes four components: instruction, model, rehearsal, and feedback (Leaf et al., 2015). This protocol can be used to train staff on observable performance-based skills that can mitigate their implicit biases. The model, rehearsal, and feedback aspects of BST are often missing from the usual didactic instruction around implicit bias. Hillard and colleagues (2013) spoke to the fact that instruction alone was not sufficient to make long lasting change in this area. With the addition of modeling, role play, and reinforcement for target behaviors such as prosocial verbal behavior towards individuals of all 
races, the staff being trained would not only learn about their implicit biases, but also how to engage in behavior that counteracts them. A recent study suggested that BST can be enhanced with the addition of ACT when training trainers on the implementation of BST (Little et al., 2020).

ACT is a methodology within the behavior analytic field, which has been used to address private and public events. When ACT is used as a therapeutic tool, the "T" corresponds to therapy. However, when used as a training method within organizations in a nonclinical manner, as it is typically used in the field of ABA, the "T" corresponds to training (Moran, 2011). Private verbal behavior can lead to avoidance of triggering situations, which may then lead to undesirable overt behavior. Furthermore, individuals may be engaging in overt behavior that is not in line with the individual's private verbal behaviors or values as related to race relations. Self-evaluation around these private verbal behaviors may be an effective part of an intervention. Regarding racial implicit bias training, the goal is to inform around biases so as to increase positive social interactions between individuals of different races. If related private events become aversive, the individual may avoid others of different races, and therefore, create, rather than solve, a problem. The six components of ACT (i.e., values, present moment attention, acceptance, defusion, self as context, and committed action) can be used to teach individuals to self-evaluate by becoming mindful and aware of their biases and thoughts, accept (rather than avoid) private events or the occurrence of challenging situations, and engage in behaviors that align with their values regarding interaction with people of different races.

Lillis and Hayes (2007) compared a traditional prejudice awareness training to the use of ACT in the college classroom setting. The students completed a questionnaire before and after each training that measured the students' intentions to engage in positive behavior related to 
prejudicial biases. This study found that the ACT instruction decreased prejudiced thoughts and increased awareness of these types of thoughts. The authors posited that this awareness came from the students' ability to understand the process by which judgments are made, regardless of whether or not these thoughts were related to race. This study identified a way to measure intention of positive behavior and opened the possibility for further discussion and research on observable behavior changes related to biases as a result of using ACT.

Kanter and colleagues (2020) focused on teaching mindfulness and acceptance skills rather than on implicit bias directly. After lecture-based training on microaggressions, biases and how they relate to patient care, the participants were given training on ACT mindfulness strategies. Of particular interest is that the participants were paired in interracial dyads and engaged in relationship building activities. This seems to have increased rapport between the dyads by pairing them with reinforcement. The final component of the training package included a rehearsal and feedback portion, in line with BST. They measured and coded verbal behavior during 10-minute interactions between the medical professional and the contrived patient, both before and after the workshop intervention. They found that the workshop intervention package resulted in decreases in offensive microaggressions and significant increases in emotional rapport building and responsiveness behaviors in the group of white medical providers. This improvement in the emotional rapport building may be significant in establishing that the individual's private verbal behavior regarding race may be impacting their overt behavior. Similarly, there is some research that suggests that medication may result in a general reduced level in implicit bias. In particular, Propranolol, which is used for the reduction of reported anxiety, also led to responses indicative of reduced implicit biases when compared to the placebo group (Terbeck et al., 2012). While more research is needed in this area, this finding does 
suggest a link between an anxiety response and behaviors related to implicit biases. It is therefore also interesting that ACT has been a suggested treatment for both. ABA can therefore lean on ACT studies for development of trainings as they provide us with a substantial model on how to impact immediate change in direct interactions with clients and caregivers.

An even simpler adjustment that a clinician can execute with urgency is the targeting of generalization of stimuli used in direct service delivery, specifically, the materials available to verbal behavior and early learner programs. The materials used by clinicians may be a sign of their implicit biases. As such, consciously incorporating more diverse stimuli is a concrete change that clinicians can make to increase their rate of anti-bias behavior. Not only will this have an impact on their individual clients, but this behavior may serve as a model for other staff members the clinician comes in contact with. Matsuda and colleagues (2020) highlighted that biases are difficult to change because of the substantial learning history, which may interfere with forming new associations or stimulus equivalence classes. If a clinician presents varied stimuli to patients when they are young, this may be key because younger individuals have not yet had substantial learning histories, potentially allowing for the influence of new learning histories in a way that limits the formation of negative implicit biases. Presenting new stimuli may result in the establishment of new relations that may alter initial relations and biases; thus, strategies can be implemented at any age. One method to accomplish this is through the use of multiple exemplar training and the inclusion not only of exemplars of the individual's own race, but those of other races as well. Multiple exemplar training has been successfully implemented to produce stimulus and response generalization across various skills and conditions (LaFrance \& Tarbox, 2020). This one small step is by no means sufficient, but it is an actionable step that can be easily and immediately enacted. 
Implicit Bias 30

\section{Discussion}

It is damaging to acknowledge the existence of implicit biases and stop there. Clinicians should assume personal accountability to actively address these implicit biases, which all individuals hold as a result of their past experiences. However, it is sometimes difficult to know what positive change can be made as individuals on this topic. It is helpful to have guidance and thus included are specific, actionable tasks that can be implemented on an organization-wide level and by an individual to affect change. The start is to consider implicit bias not as automatic nor solely as a private event, but rather as a set of behaviors that can be changed. This can result in positive outcomes, not only for individuals of a particular race, but rather for all staff and clients. With this paper, the hope is to present an initial accounting of measurable behaviors that can be assessed and interventions within the scope of ABA that can be implemented to address implicit bias. Reinforcement, self-management, BST, ACT, and generalization of stimuli are an initial set of interventions, and as research is continued in this area, more can be identified.

There are several limitations of this paper. First and foremost, evidence is not available to connect the majority of the behaviors that could be assessed to measures of implicit bias (e.g., IAT, IRAP). While it is important that future research evaluates this connection, lack of evidence should not stop clinicians from striving to engage in more inclusive behaviors now. Regardless of the link between specific behaviors and current measures of implicit bias, the identification and reduction of behaviors that are differentially applied to clients of various races is critical to providing equitable care for all clients. Additionally, while some of the strategies listed have empirical support for reducing implicit bias, others do not; nevertheless, all of the listed strategies have been researched and identified as effective in improving other behaviors. Additionally, implementation of the above recommendations likely will be challenging for 
clinicians to do on a continual basis and not as merely a one-time performative change. The clinician's ability to make ongoing change may depend on the resources of their workplace, competing contingencies on their time, and their own ability to self-assess, especially in the presence of stressors. The presence (or lack thereof) of another individual to aid in gathering and reviewing data may also impact the clinician's ability to use these strategies. However, to reduce the potential harm caused by implicit bias, it is critical for clinicians to assess and modify their own practices as soon as possible; the above-listed strategies are an appropriate starting place for clinicians. In the absence of evidence, data should be collected on an individual basis, with clinicians making data-based decisions.

In order to focus the behaviors and strategies discussed, this paper concentrated on race, as opposed to other potential sources of bias. As other sources of bias are critical to address in society, and in the work provided by ABA clinicians, future research should address these additional areas. Furthermore, this paper focused on implicit biases of individual clinicians. Implicit bias can certainly operate on a group level, and while it was beyond the scope of this paper, future research on the intervention of group implicit biases is warranted.

Additionally, this paper provides examples of measurable behaviors that may be related to implicit bias; this is not a comprehensive list. The goal of this paper is to encourage critical thinking on the part of clinicians. As such, clinicians are encouraged to focus on the larger picture; reducing one small behavior while still engaging in a pattern of implicit bias is insufficient. However, making numerous small changes in behavior toward a larger goal have the potential to positively impact client care and staff interactions. Social validity assessments are one potential way to ensure that clinicians are focused on the most relevant areas. 
The goal of this paper was to identify actionable steps to make meaningful change in reducing the more subtle acts of discrimination that clients may experience and increasing inclusive practices. While the focus of this paper is on actions clinicians can take, researchers also play an important role in reducing implicit bias in our field. Not only should researchers increase reports on the diversity of populations in studies, but also, they should include individuals from a variety of races to aid the generalizability of results. Additionally, researchers in the field of ABA should include studies specifically on topics such as implicit bias, diversity, and racism. While the field of ABA has much to offer in these areas, scant attention has been paid to them.

The field of behavior analysis has many tools to effect behavior change; using these tools to address implicit bias can improve our effectiveness as clinicians and as supervisors of staff. As awareness in the area of implicit bias expands, it is critical to explore the interplay of race and culture. As stated by Miller and colleagues (2019), "What we need to learn is not about the habits and preferences of culture $\mathrm{X}$ but rather how to learn about learning about the cultures of others and to be open to constantly changing conditions that may very often be different from our own experiences." Explicitly analyzing and discussing the impact of race as well as culture is an important step to reducing discrimination.

As this paper is just one step toward addressing racism, the onus is now on clinicians to be open to exploring these topics and continuing this discussion. What is most needed in the field of $\mathrm{ABA}$ at this time is self-reflection and openness to changes. The area of implicit bias is a work in progress; as more individuals and researchers share their perspectives, a fuller understanding will grow. While those reading this paper likely share the value of reducing racial implicit bias in their practice, it is likely that a subset of ABA clinicians exist who do not share 
Implicit Bias 33

these values. Thus, it is critical for clinicians who value racial equity to share information about bias and discrimination with colleagues and connect it to the Professional and Ethical Compliance Code for Behavior Analysts (BACB, 2014) and client outcomes. ABA clinicians should continue to explore the perspectives of others and learn about the history of other social groups to which their clients belong.

Ultimately, the goal is to provide improved care and seek better outcomes for all clients and staff, not just those who are of the same race as the clinician they work with. ABA clinicians must start by assessing values, both personal and organizational. A drive to be inclusive is in line with the values that may have brought clinicians to the field (e.g., a desire to help and demonstrate compassion). For clinicians working with individuals from cultures and races other than their own, identifying and addressing any incongruity between their behaviors and their values is a critical step in reducing implicitly biased behaviors. Using the language modeled from ACT, what is currently missing, and what should be the next step, is committed action. While taking steps to analyze their actions with clients and staff may be uncomfortable for clinicians, now is the time for clinicians to sit with their discomfort in order to make changes to ensure equitable care is provided to all clients and staff.

Conflict of Interest: The authors declare that they have no conflict of interest.

Ethical approval: This article does not contain research conducted with human participants. 
Implicit Bias 34






\section{References}

Barnes-Holmes, D., Murphy, A., \& Barnes-Holmes, Y. (2010). The implicit relational assessment procedure: Exploring the impact of private versus public contexts and the response latency criterion on pro-white and anti-Black stereotyping among white Irish individuals. The Psychological Record, 60, 57-79. https://doi.org/10.1007/BF03395694

Behavior Analyst Certification Board. (n.d). BACB certificant data. Retrieved from https://www.bacb.com/BACB-certificant-data.

Behavior Analyst Certification Board. (2014). Professional and ethical compliance code for behavior analysts. Retrieved from https://www.bacb.com/wp-content/uploads/BACBCompliance-Code-english_190318.pdf

Bernhard, B. (2019, October 1). Autism insurance coverage now required in all 50 states. Disability Scoop. https://www.disabilityscoop.com/2019/10/01/autism-insurancecoverage-now-required-50states/27223/\#: :text=After\%20officials\%20in\%20the\%20last,for\%20the\%20treatment \%20of\%20autism.

Brookman-Frazee, L. (2004). Using parent/clinician partnerships in parent education programs for children with autism. Journal of Positive Behavior Interventions, 6(4), 195-213. https://doi.org/10.1177/10983007040060040201

Capell, S. T., \& Sevon, M. A. (2020). Applied behavior analysis within the African American community. In B. M. Conners \& S. T. Capell (Eds.), Multiculturalism and diversity in applied behavior analysis: Bridging theory and application (pp. 29-46). Routledge. 
Critchfield, T. S., Barnes-Holmes, D., \& Dougher, M. J. (2018). Editorial: What Sidman did-Historical and contemporary significance of research on derived stimulus relations. Perspectives on Behavior Science, 41, 9-32. https://doi.org/10.1007/s40614-018-0154-9

De Houwer, J. (2019). Implicit bias is behavior: A functional-cognitive perspective on implicit bias. Perspectives on Psychological Science, 14(5), 835-840. https://doi.org/10.1177/1745691619855638

Derman-Sparks, L. \& the A.B.C. Task Force. (2001). Anti-bias curriculum: Tools for empowering young children. National Association for the Education of Young Children.

Devine, P. G., Forscher, P. S., Austin, A. J., \& Cox, W. T. L. (2012). Long-term reduction in implicit race bias: A prejudice habit-breaking intervention. Journal of Experimental Social Psychology, 48(6), 1267-1278. https://doi.org/10.1016/j.jesp.2012.06.003

Dixon, M. R., Dymond, S., Rehfeldt, R. A., Roche, B., \& Zlomke, K. R. (2003). Terrorism and relational frame theory. Behavior and Social Issues, 12(2), 129-147. https://doi.org/10.5210/bsi.v12i2.40

Drake, C. E., Kramer, S., Sain, T., Swiatek, R., Kohn, K., \& Murphy, M. (2015). Exploring the reliability and convergent validity of implicit racial evaluations. Behavior and Social Issues, 24, 68-87. https://doi.org/10.5210/bsi.v24i0.5496

Drysdale, H., van der Meer, L., \& Kagohara, D. (2015). Children with autism spectrum disorder from bilingual families: A systematic review. Review Journal of Autism and Developmental Disorders, 2, 26-38. http://dx.doi.org/10.1016/j.jcomdis.2016.07.003

FitzGerald, C. \& Hurst, S. (2017). Implicit bias in healthcare professionals: A systematic review. BMC Medical Ethics, 18(19). https://doi.org/10.1186/s12910-017-0179-8 
Fernandes, R. (2016). How bias training works in one campus police department. The Chronicle of Higher Education. Retrieved from https://search.proquest.com/docview/1790790500?accountid=166077

Fong, E. H., Catagnus, R. M., Brodhead, M. T., Quigly, S., \& Field, S. (2016). Developing the cultural awareness skills of behavior analysts. Behavior Analysis in Practice, 9, 84-94. https://doi.org/10.1007/s40617-016-0111-6

Fong, E. H., Ficklin, S., \& Lee, H. Y. (2017). Increasing cultural understanding and diversity in applied behavior analysis. Behavior Analysis: Research and Practice, 17(2), 103-113. https://doi.org/10.1037/bar0000076

Hillard, A. L., Ryan, C. S., \& Gervais, S. J. (2013). Reactions to the implicit association test as an educational tool: A mixed methods study. Social Psychology of Education, 16, 495516. https://doi.org/10.1007/s11218-013-9219-5

Jackson, J.L. (2018). The non-performativity of implicit bias training. Radical Teacher, 112, 4654.

Kanter, J. W., Rosen, D.C., Manbeck, K. E., Branstetter, H. M., Kuczynski, A. M., Corey, M. D., Maitland, D. W. M., \& Williams, M. T. (2020). Addressing microaggressions in racially charged patient-provider interactions: A pilot randomized trial. BMC Medical Education, 20(88). https://doi.org/10.1186/s12909-020-02004-9

Kempf, A. (2020). If we are going to talk about implicit race bias, we need to talk about structural racism: moving beyond ubiquity and inevitability in teaching and learning about race. Taboo: The Journal of Culture and Education, 19(2), 115-132. Retrieved from https://digitalscholarship.unlv.edu/taboo/vol19/iss2/10 
Kim, D. (2003). Voluntary controllability of the Implicit Association Test (IAT). Social Psychology Quarterly, 66(1), 83-96. https://doi.org/10.2307/3090143

Knochel, A. E. (2019). Bridging the achievement gap: Promoting equity in teachers' use of behavior-specific praise with self-monitoring and performance feedback. [Graduate Theses and Dissertations, University of South Florida]. Scholar Commons. https://scholarcommons.usf.edu/etd/7831

Kurdi, B., Seitchik, A. E., Axt, J. R., Carroll, T. J., Karapetyan, A., Kaushik, N., Tomezsko, D., Greenwald, A. G., \& Banaji, M. R. (2018). Relationship between the Implicit Association Test and intergroup behavior: A meta-analysis. American Psychologist, 74(5), 569-586. https://doi.org/10.1037/amp0000364

LaFrance, D. L. \& Tarbox, J. (2020). The importance of multiple exemplar instruction in the establishment of novel verbal behavior. Journal of Applied Behavior Analysis, 53(1), 1024. https://doi.org/10.1002/jaba.611

Lai, C. K., Hoffman, K. M., \& Nosek, B. A. (2013). Reducing implicit prejudice. Social and Personality Psychology Compass. 7(5), 315-330. https://doi.org/10.1111/spc3.12023

Lang, R., Rispoli, M., Sigafoos, J., Lancioni, G., Andrews, A., \& Ortega, L. (2011). Effects of language of instruction on response accuracy and challenging behavior in a child with autism. Journal of Behavioral Education, 20, 252-259. https://doi.org/10.1007/s10864$011-9130-0$

Leaf, J. B., Townley-Cochran, D., Taubman, M., Cihon, J. H., Oppenheim-Leaf, M. L., Kassardjian, A., Leaf, R., McEachin, J., \& Pentz, T. G. (2015). The teaching interaction procedure and behavioral skills training for individuals diagnosed with autism spectrum 
disorder: A review and commentary. Review Journal of Autism and Developmental Disorders, 2, 402-413. https://doi.org/10.1007/s40489-015-0060-y

Lillis, J. \& Hayes, S. C. (2007). Applying acceptance, mindfulness, and values to the reduction of prejudice: A pilot study. Behavior Modification, 31(4), 389-411. https://doi.org/10.1177/0145445506298413

Lim, N. \& Charlop, M. H. (2018). Effects of English versus heritage language on play in bilingually exposed children with autism spectrum disorder. Behavioral Interventions, 33(4), 339-351. https://doi.org/10.1002/bin.1644

Little, A., Tarbox, J., \& Alzaabi, K. (2020). Using acceptance and commitment training to enhance the effectiveness of behavioral skills training. Journal of Contextual Behavioral Science, 16, 9-16. https://doi.org/10.1016/j.jcbs.2020.02.002

Magaña, S., Parish, S. L., Rose, R. A., Timberlake, M., \& Swaine, J. G. (2012). Racial and ethnic disparities in quality of health care among children with autism and other developmental disabilities. Intellectual and Developmental Disabilities, 50(4), 287-299. https://doi.org/10.1352/1934-9556-50.4.287

Matsuda, K., Garcia, Y., Catagnus, R., \& Brandt, J. A. (2020). Can behavior analysis help us understand and reduce racism? A review of the current literature. Behavior Analysis in Practice, 13, 336-347.https://doi.org/10.1007/s40617-020-00411-4

Meissner, F., Grigutsch, L. A., Koranyi, N., Müller, F., \& Rothermund, K. (2019). Predicting behavior with implicit measures: Disillusioning findings, reasonable explanations, and sophisticated solutions. Frontiers in Psychology, 10(2483). https://doi.org/10.3389/fpsyg.2019.02483 
Miller, K. L., Re Cruz, A., \& Ala'i-Rosales, S. (2019). Inherent tensions and possibilities: Behavior analysis and cultural responsiveness. Behavior and Social Issues, 28, 16-36. https://doi.org/10.1007/s42822-019-00010-1

Moran, D.J. (2011). ACT for leadership: Using Acceptance and Commitment Training to develop crisis-resilient change managers. The International Journal of Behavioral Consultation and Therapy, 7(1), 66-75. http://dx.doi.org/10.1037/h0100928

Power, P., Barnes-Holmes, D., Barnes-Holmes, Y., \& Stewart, I. (2009). The Implicit Relational Assessment Procedure (IRAP) as a measure of implicit relative preferences: A first study. The Psychological Record, 59, 621-640. https://doi.org/10.1007/BF03395684

Roche, M. J. L., Bush, H. H., \& D’Angelo, E. (2018). The assessment and treatment of autism spectrum disorder: A cultural examination. Practice Innovations, 3(2), 107-122. https://doi.org/10.1037/pri0000067

Schultz, P. L. \& Baker, J. (2017). Teaching strategies to increase nursing student acceptance and management of unconscious bias. Journal of Nursing Education, 56(11), 692-696. https://doi.org/10.3928/01484834-20171020-11

Steffens, M. C. (2004). Is the Implicit Association Test immune to faking? Experimental Psychology, 51(3), 165-179. https://doi.org/10.1027/1618-3169.51.3.165

Tagg, R. (2020). ADDRESSING cultural complexities. In B. M. Conners \& S. T. Capell (Eds.), Multiculturalism and diversity in applied behavior analysis: Bridging theory and application (pp. 5-18). Routledge.

Taylor, B. A., LeBlanc, L. A., \& Nosik, M. R. (2019). Compassionate care in behavior analytic treatment: Can outcomes be enhanced by attending to relationships with caregivers? Behavior Analysis in Practice, 12, 654-666. https://doi.org/10.1007/s40617-018-00289-3 
Terbeck, S., Kahane, G., McTavish, S., Savulescu, J., Cowen, P. J., \& Hewstone, M. (2012). Propranolol reduces implicit negative racial bias. Psychopharmacology, 222, 419-424. https://doi.org/10.1007/s00213-012-2657-5

U.S. Census Bureau (2019). QuickFacts. https://www.census.gov/data/data-tools/quickfacts.html

U.S. Department of Justice, Federal Bureau of Investigation (2020). Hate crime statistics, 2019. https://ucr.fbi.gov/hate-crime/2019

Zestcott, C. A., Blair, I. V., \& Stone, J. (2016). Examining the presence, consequences, and reduction of implicit bias in health care: A narrative review. Group Processes and Intergroup Relations, 19(4), 528-542. https://doi.org/10.1177/1368430216642029

Zuckerman, K. E., Lindly, O. J., Reyes, N. M., Chavez, A. E., Macias, K., Smith, K. N., \& Reynolds, A. (2017). Disparities in diagnosis and treatment of autism in Latino and nonLatino white families. Pediatrics, 139(5). https://doi.org/10.1542/peds.2016-3010 
Table 1

Assessment of Implicit Bias for Clinicians

Context/Item Operational Definition(s) Examples

Intake

Demographic information on race/culture

Questions about cultural preferences

Acknowledgment of cultural preferences
Number of items that address culture/race in intake paperwork

Asks open-ended questions instead of closed-ended questions; asks relevant questions about aspects of the family's culture; number of questions asked by the clinician

Avoids judgmental statements; responds supportively; follows up on concerns
Intake questionnaire item that asks the client/caregiver to identify their race/ethnicity

"What does bedtime look like for your family?"; "Who serves as a caregiver for your child?"; "What are your expectations for eye contact for your child?"

A clinician discusses pre-requisites and how to address the skill when a caregiver expresses desire for their child who currently uses one-word sentences to be polite (e.g., use please and thank you) 
Treatment planning

Client Interactions

Vocal behavior

Non-vocal behavior

Stimuli
Frequency that input from the client/caregiver is sought regarding the inclusion of goals/strategies within the treatment plan and behavior intervention plan

Makes statements of praise; provides corrections/redirections; rate of speech; tone of voice

Use of restraint or other physical procedures; makes eye contact; displays welcoming facial expressions and body language; frequency and duration of physical reinforcers

Includes client's race and other diverse backgrounds; excludes stereotypic images
"Would you like the treatment plan to address your child sleeping in their own bed?"; "I noted that your child isn't feeding themselves yet, is this something you want to prioritize working on?"; "One option to address your child's aggression is to ignore the behavior, is that something you're open to?"

Praise statement: "I liked how you said hi to your friends today." Correction: "Let's try that again."

Use of restraint is tracked for all clients and analyzed based on client race

Assess a client's visuals to identify what percentage include members of different races 
Language of instruction

\section{Response to explicit racial challenges}

\section{Caregiver Interactions}

Verbal microaggressions

Assuming instead of asking
Incorporates client's native language

Responds to questions factually; does not ignore, side-step, or criticize the question.

Makes overt or subtle statements pertaining to race that are rude or demeaning

Number of questions asked by the clinician
For each client whose native language is not English, the clinician notes if and how their native language is used during intervention

See Table 2 for example scoring system based on Kanter et al., 2020

"Your English is really good!"; "You're so wellspoken"; "Where were you born?"; "I don't see color"; continually mispronouncing client name after correction

Assumption: Clinician assumes that the client's father isn't a part of the client's life and doesn't ask any questions about the father. Question (to the mother): "How involved is your child's father in their care?" 
Reinforcing/positive statements

Collaborative versus authoritative strategies

Consideration of extended family members

Language of preference
Number of positive statements

Provides choices; asks open-ended questions; makes suggestions instead of directives; involves the caregiver in decision making

\section{Considers views and values of extended family members}

Asks about language preference; offers translator services
"I'm so glad to hear that you provided your child with choices!" "That's great that you made the opportunity to work in the conversation skills we've discussed.'

Collaborative: "To teach your child to get your attention, we can work on them tapping you or using a device, which would you prefer?" Authoritative: "I'm going to add a lesson to the program to teach your child to tap to gain attention."

"Are there other family members whose opinion you value, in terms of making decisions for your child's treatment?"

"We do have the ability to use a translator - what language do you prefer for our meetings?" 
Training

Use of evidence-based strategies (e.g., behavioral skills training)

Staff Interactions

Training
Representation of diversity in training staff; supervisor's disciplinary and supportive actions toward staff; social validity on perception of equitable provision of training opportunities
Tracking data on the use of the four components of behavioral skills training (i.e., instruction, modeling, rehearsal, feedback).

Tracking opportunity data on supervisor's reactions to staff asking questions (e.g., did the supervisor ignore or follow up supportively); providing all staff with a social validity questionnaire to gather data on their perception of the training opportunities they were presented with 
Mentorship

Development of professional
goals

Assess professional goals based on their function, social significance, the specific behavior targeted, why it is targeted, and the impact of that change
A mentor reviewing notes (e.g., permanent product data) to assess the frequency with which she discussed race with each of her mentees

All professional goals within a center are reviewed and coded based on function and social significance, then analyzed based on supervisee race 
Table 2

Responsiveness to Racial Challenges Scale, Adapted from Kanter et al. (2020)

\begin{tabular}{|c|c|c|c|c|c|}
\hline \multirow[b]{3}{*}{ Example Scenario } & \multicolumn{5}{|c|}{ Score and Description } \\
\hline & & $\begin{array}{l}-1 \text { : Mild Negative } \\
\text { Reaction/Ignoring }\end{array}$ & 0: Passive Reaction & $\begin{array}{l}\text { 1: Mild Positive } \\
\text { Reaction }\end{array}$ & $\begin{array}{l}\text { 2: Strong Positive } \\
\text { Reaction }\end{array}$ \\
\hline & $\begin{array}{l}-2: \text { Strong Negative } \\
\text { Reaction }\end{array}$ & & & & \\
\hline $\begin{array}{l}\text { The client says, } \\
\text { "Why does the } \\
\text { band-aid match } \\
\text { your skin but not } \\
\text { mine?" }\end{array}$ & $\begin{array}{l}\text { "If your skin was } \\
\text { beige, then it } \\
\text { would match the } \\
\text { band-aid." }\end{array}$ & $\begin{array}{l}\text { "That's the way } \\
\text { band-aids are." }\end{array}$ & $\begin{array}{c}\text { "It sounds like } \\
\text { that's a bit } \\
\text { confusing." }\end{array}$ & $\begin{array}{l}\text { "That's frustrating, } \\
\text { it would be nice } \\
\text { if band-aids } \\
\text { came in your } \\
\text { skin tone too!" }\end{array}$ & $\begin{array}{l}\text { "I think the people } \\
\text { who make band- } \\
\text { aids have this } \\
\text { kind of skin tone } \\
\text { and they didn't } \\
\text { think about } \\
\text { others with } \\
\text { different skin } \\
\text { colors. That's } \\
\text { really not okay." }\end{array}$ \\
\hline
\end{tabular}


Another child says, "Why is his hair long?" have that long

hair."
"People like him all
"That's just how his hair is."
"I like his hair that way."
Different people have different kind of hair, and that's okay!"

\section{Caregiver says, \\ "We live in the \\ Black part of \\ town, so our \\ school services \\ aren't great."}

$\begin{array}{cc}\text { "Well, that's just } & \text { "What are your } \\ \text { what happens } & \text { child's school } \\ \text { when you } & \text { services like?" }\end{array}$

choose to live in

that area."
"You think the services are poor
because of
where you
live?"
"That must be really
frustrating!"

"People are all different and they're born with different types of hair. No type of hair is better or worse than another, they are all beautiful!"

“I'm sorry to hear that. It's not okay when systemic racism impacts the schooling our children receive." 
Staff member says, "I'll never learn how to

pronounce this

client's name, I

wish her parents

picked an easier

name for her."
"I know! It's such

an odd name."
"Yeah, I agree."

"Yeah, I agree."
"Maybe her parents like that name."
"Parents can pick whatever name they want for their children!'

"It's not our place to judge other's names. We should make an effort to learn the right pronunciation."

Note: Example clinician responses are provided for each score. Adapted from Addressing Microaggressions in Racially Charged Patient-Provider Interactions: A Pilot Randomized Trial,” by J. W. Kanter, D. C. Rosen, K. E. Manbeck, H. M. Branstetter, A. M. Kuczynski, M. D. Corey, D. W. M. Maitland, and M. T. Williams, 2020, BMC Medical Education, 20(88), p. 6 (https://doi.org/10.1186/s12909-020-02004-9). Copyright 2020 by the authors. 
Table 3

Interventions to Reduce Implicit Bias for Clinicians

\begin{tabular}{lll}
\hline Intervention & Suggested Applications & Research \\
\hline Reinforcement & Intake: Equitable provision of open-ended questions and interventions that & Knochel (2019) \\
stem from client/caregiver input. & Clinician-Client Interactions: Equitable delivery of reinforcers. \\
& Clinician-Caregiver Interactions: Equitable use of collaborative strategies. \\
& Clinician-Staff Interactions: Equitable access to training or mentoring \\
& opportunities. Increasing opportunities for relationship building activities \\
& between interracial staff. \\
& \\
Self- & \\
Management & Intake: Monitor number of questions about cultural preferences asked. & Knochel (2019), Schultz \& \\
Clinician-Client Interactions: Monitor praise and corrective statements used. & Baker (2017) \\
Clinician-Caregiver Interactions: Monitor use of collaborative strategies or \\
positive phrases. \\
Clinician-Staff Interactions: Monitor discussions of race during mentorship \\
opportunities.
\end{tabular}


Behavioral skills training (BST)

Acceptance and commitment training (ACT)

\section{Multiple exemplar training}

Clinician-Staff Interactions: Use BST to train staff in responding to racial challenges, increasing pro-social behaviors (e.g., using praise statements), or asking questions of caregivers instead of making assumptions.

Clinician-Caregiver Interactions: Use of collaborative strategies.

Clinician-Staff Interactions: Increasing awareness around prejudice thoughts. Reducing use of microaggressions.

Clinician-Client Interactions: Inclusion of different races in instructional material for clients.

Clinician-Staff Interactions: Inclusion of different races in training materials for staff. Diversity in training staff, as well in managerial or corporate staff.
Leaf et al., (2015), when paired with ACT - (Little et al., 2020)

Lillis and Hayes (2007), Kanter and colleagues (2020)

LaFrance \& Tarbox, (2020) 